\section{Uso de terapia antirretroviral de gran actividad (TARGA) y transmisión vertical del VIH. Cuba 1989-2008}

Tania Roig Álvarez 1

Ida González Núñez 2

Dennis Verdasquera Corcho 3

\section{Use of highly active antiretroviral therapy (HAART) and HIV vertical transmission. Cuba 1989-2008}

1 Hospital Ginecobstétrico Docente Ramón González Coro. Calle 21 \# 855 entre 4 y 6. Plaza de la Revolución. La Habana. Cuba. E mail: tania.roig@infomed.sld.cu

2,3 Instituto de Medicina Tropical Pedro Kouri. Avenida Novia del Mediodía KM $6 \frac{1}{2}$ La Lisa. La Habana, Cuba.

\begin{abstract}
Objectives: to describe the use of Highly Active Antiretroviral Therapy (HAART) in HIV-positive Cuban pregnant women and the relationship between the type of mother antiretroviral treatment used during pregnancy and perinatal transmission.

Methods: a cross-sectional study was carried out in 319 children from seropositive mothers, identifying in they HIV infections or not. The descriptive measures, the rate comparative test and the prevalence ratio were used for the analysis.

Results: the overall rate of mother-to-child transmission was 5\% and this was higher in the years in which HAART were not used $(9,5 \%$ vs $3,1 \%$; $p=0,049)$. Initially, only that women classified as AIDS received combinative therapy. As time passed, an increased use of HAART and a decreased rate of vertical transmission was observed (26,3\% in 2003 vs $86,7 \%$ in 2008 and $10,5 \%$ in 2003 vs 2,1\% in 2008; respectively). The prevalence of the disease decreased with the use of a more active maternal treatment (lineal tendency $p=0,017$ ).

Conclusions: the improvement of Cuban prevention protocol decreased HIV vertical transmission. Key words HIV infections, Pregnancy
\end{abstract}

\section{Resumo}

Objetivos: describir el uso de terapia antirretroviral de gran actividad (TARGA) en gestantes cubanas infectadas por el VIH y el comportamiento de la transmisión vertical del VIH, según tratamiento antirretroviral materno usado durante la gestación.

Métodos: se realizó un estudio de corte transversal donde se incluyeron 319 niños hijos de mujeres seropositivas al VIH, identificándose en ellos la presencia de infección o no por el VIH; para el análisis se utilizaron medidas descriptivas, la prueba de comparación de tasas y la razón de prevalencia.

Resultados: la transmisión vertical del VIH de forma general fue de 5\% y esta fue mayor en los años en que no se usaba TARGA (9,5\% vs 3,1\%; p=0,049). Inicialmente solo recibian TARGA gestantes clasificadas como sida. En el transcurso del tiempo se observó un incremento del uso de este esquema de tratamiento en gestantes y una disminución en la infección de la descendencia (26,3\% en 2003 vs $86,7 \%$ en 2008 y $10,5 \%$ en 2003 vs $2,1 \%$ en 2008; respectivamente). La prevalencia de la enfermedad disminuyó al usar un esquema de tratamiento materno de mayor actividad (tendencia lineal $p=0,017)$.

Conclusiones: el perfeccionamiento del programa de prevención cubano ha disminuido la transmisión vertical del VIH.

Palavras-chave Infecciones por VIH, Embarazo 


\section{Introducción}

Con el uso de terapia antirretroviral de gran actividad (TARGA), la suspensión de la lactancia materna, la realización de cesárea, y la determinación de resistencia del virus de inmunodeficiencia humana (VIH) a los antirretrovirales en todas las gestantes sin tratamiento previo o las que tenían tratamiento antes del embarazo, con evidencia de falla del mismo; se obtienen disminuciones significativas de la transmisión del VIH de madre a hijo $(1-2 \%)$.

En la actualidad se preconiza el uso de TARGA a todas las gestantes sin tratamiento previo, independientemente de la carga viral que presenten. La combinación de zidovudina (AZT), lamivudina más lopinavir/ritonavir o nevirapina es el régimen preferido, este último no debe ser usado de primera línea en mujeres con conteo de linfocitos $\mathrm{T} \mathrm{CD}_{4}{ }^{+}>$ 250 células $/ \mathrm{mm}^{3}$ debido al riesgo potencial de toxicidad hepática. 2,3

En Cuba en el año 1986 se implementa el Programa Nacional de Prevención y Control del VIH/SIDA, cuya primera medida profiláctica, relacionada con la transmisión vertical fue la suspensión de la lactancia materna en hijos de madres infectadas por el virus. En 1989 se introdujo la indicación del parto por cesárea; en 1997, la profilaxis con AZT oral a la madre durante la gestación y hasta el momento del parto y al neonato, también por vía oral, durante las primeras seis semanas de vida. $4,5 \mathrm{~A}$ partir de Julio del año 2002 se orientó TARGA a todas las gestantes con conteo de linfocitos $\mathrm{T} \mathrm{CD}_{4}{ }^{+}<$ 350 células $/ \mathrm{mm}^{3}$ y carga viral $>1000$ copias $/ \mathrm{mL}$, sin terapia previa 6 y a inicios del año 2005 se comenzó el uso de AZT intraparto endovenosa, con lo cual se completó las tres partes de AZT, que recomienda el protocolo ACTG 076.7

A finales del año 2008 se hizo nuevamente una revisión del programa de prevención de la transmisión vertical y teniendo en consideración las últimas recomendaciones a nivel mundial, se decidió indicar TARGA que incluya el AZT de ser posible, a todas las gestantes independientemente del conteo de linfocitos $\mathrm{T} \mathrm{CD}_{4}^{+}$y la carga viral y reevaluar su retirada a los dos meses postparto en dependencia de la valoración clínica, virológica e inmunológica en ese momento. En el último trimestre del año 2011 se decidió no retirar el tratamiento después del parto a las mujeres que iniciaron terapia durante la gestación

Se decide realizar una investigación con el objetivo de describir el uso de TARGA en gestantes cubanas infectadas por el VIH y el comportamiento de la transmisión vertical del VIH, según tratamiento antirretroviral materno usado durante la gestación.

\section{Métodos}

Se realizó un estudio de corte transversal donde se incluyeron a 319 niños hijos de mujeres seropositivas al VIH, nacidos en Cuba del 1 ro de Enero de 1989 al 31 de Diciembre del 2008 que cumplieron los criterios de inclusión siguientes:

- madre infectada con el VIH-1

- diagnóstico de la infección materna antes o durante el embarazo del hijo en cuestión

- incluido en la base de datos de la consulta especializada de pediatría del Instituto de Medicina Tropical Pedro Kourí (IPK)

- atendidos en la consulta especializada de pediatría, antes del cierre del año 2010 y con historia clínica en el IPK.

Se excluyeron a los hijos de mujeres seropositivas al VIH-2, a los hijos de mujeres con diagnóstico de la infección por el VIH-1 después del nacimiento del hijo en cuestión, a los niños que no tenían historia clínica del IPK y a los infantes que se atendieron en la consulta especializada de pediatría, pero su nacimiento ocurrió fuera de Cuba.

Se consideraron en este universo como niños infectados por el VIH a los 16 niños que presentaron dos estudios virológicos positivos (detección del ADN del VIH en células mononucleares de sangre periférica $\mathrm{y} / \mathrm{o}$ detección del ARN del VIH extracelular en plasma) y como sanos a los 303 niños que tuvieron dos o más estudios virológicos negativos durante los primeros 18 meses de vida y dos Western blot negativos entre los 12 y 18 meses de edad.

Los datos se recogieron de las historias clínicas de los niños y las madres atendidos en el IPK y de la base de datos del seguimiento de los hijos de seropositivas e infectados por el VIH, por vía vertical, de la consulta especializada de pediatría de dicha institución.

Para el análisis de los datos se usaron frecuencias absolutas y relativas; la prueba de comparación de tasas de incidencia; la razón de prevalencia con intervalo de confianza de Wolf, según nivel de exposición al factor de riesgo; la prueba de tendencia lineal y la prueba de homogeneidad entre niveles. El procesamiento estadístico se realizó con el programa Epidat versión $3.1^{8}$ y se usó como nivel de significación estadística el valor de $p<0,05$. 


\section{Resultados}

La transmisión vertical del VIH general fue de 5\% (16/319). Se encontraron diferencias significativas en la transmisión vertical, en niños nacidos en los años que no se indicaba TARGA en gestantes (19892002), al compararlo con los nacidos en los años en que si se indicaba este tratamiento (2003-2008): $9,5 \%(9 / 95)$ vs $3,1 \%(7 / 224) ; p=0,049$.

En la Tabla 1 se muestra el porcentaje de mujeres que recibieron TARGA durante la gestación, según año de nacimiento de sus hijos; en los años
2007 y 2008 se observa un incremento paulatino en la indicación de este tipo de tratamiento.

En las madres de cinco niños nacidos en el año 2003, es que por primera vez se reporta el uso de TARGA en gestantes en Cuba; todas estas mujeres estaban clasificadas como en etapa Sida de la enfermedad y recibían este tratamiento antes del embarazo. En el año 2004 en ocho niños se reportó el antecedente de uso de TARGA en sus madres durante el embarazo, la mayoría de ellas (87,5\%; $7 / 8$ ) recibían este tratamiento previo a la gestación.

En niños nacidos en los años 2005, 2006 y 2007,

Tabla 1

Uso de TARGA en gestantes según año de nacimiento del niño. Cuba 2003-2008.

\begin{tabular}{lccc}
\hline & & & TARGA en madres \\
\cline { 3 - 4 } Años & Nacimientos & $n$ & $\%$ \\
\cline { 3 - 4 } 2003 & 19 & 5 & 26,32 \\
2004 & 36 & 8 & 22,22 \\
2005 & 50 & 8 & 16,00 \\
2006 & 33 & 9 & 27,27 \\
2007 & 41 & 19 & 46,34 \\
2008 & 45 & 39 & 86,64 \\
Total & 224 & 88 & 39,29 \\
\hline
\end{tabular}

TARGA = terapia con tres antirretrovirales con al menos un inhibidor no nucleósido de la reversa transcriptasa o un inhibidor de proteasas.

con antecedentes de uso de TARGA durante la gestación en sus madres, la principal indicación de este tratamiento fue el conteo de linfocitos $\mathrm{T} \mathrm{CD}_{4}{ }^{+}<$ 350 células $/ \mathrm{mm}^{3} \quad \mathrm{y} / \mathrm{o}$ la carga viral $>1000$ copias $/ \mathrm{mL}(62,5 \%, 5 / 8 ; 55,6 \%, 5 / 9$ y $52,6 \%, 10 / 19$, respectivamente).

En 39 niños nacidos en el año 2008 se usó TARGA en sus madres durante el embarazo, en la mayoría de ellas $(59 \% ; 23 / 39)$ porque a finales de este año se orientó indicar este tipo de tratamiento a todas las gestantes infectadas por el VIH, independientemente del estado virológico e inmunológico (Figura 1).

En la Figura 2 se muestra el uso de TARGA y el comportamiento de la transmisión vertical según año de nacimiento del niño; observándose un incremento del uso de este esquema de tratamiento en gestantes y una disminución en la infección de la descendencia en los últimos años.

En la Tabla 2 se muestra la transmisión vertical del VIH según tipo de tratamiento usado en la madre durante la gestación; se observó la mayor transmisión en los hijos de mujeres que no recibieron tratamiento durante el embarazo $(15,56 \%)$ y la prevalencia de la enfermedad diminuyó al usar un esquema de tratamiento materno de mayor actividad (homogeneidad entre niveles $p=0,006$; tendencia lineal $p=0,017$ ). 


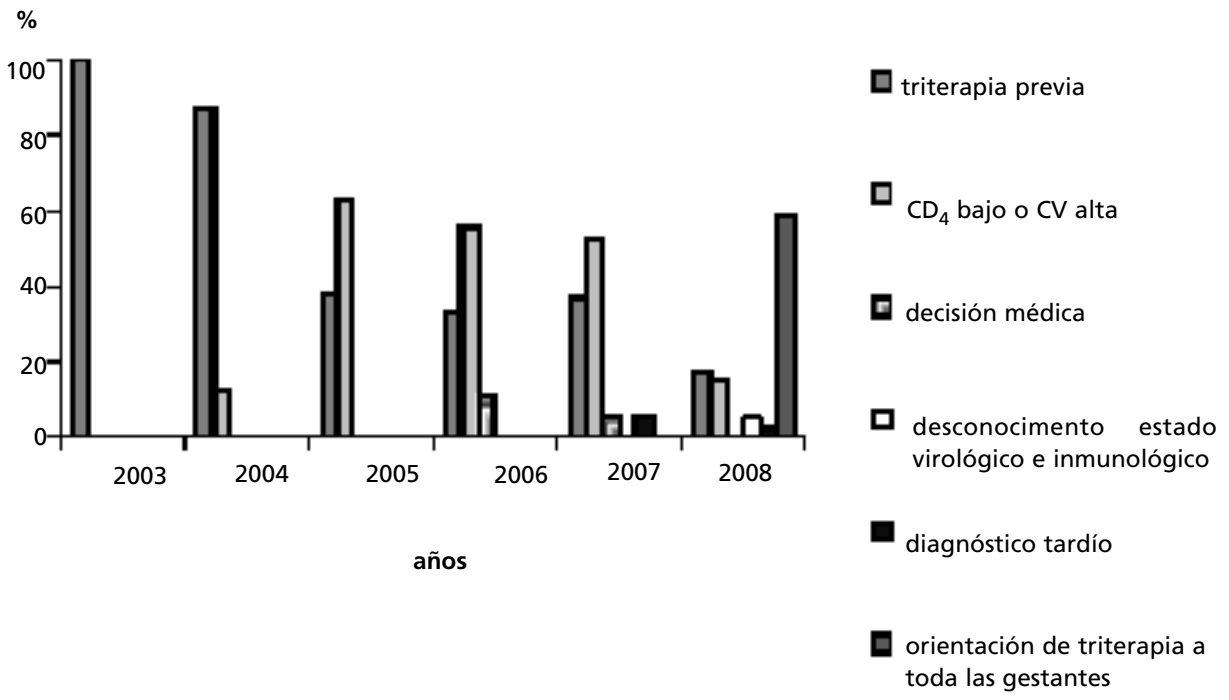

TARGA = terapia con tres antirretrovirales con al menos un inhibidor no nucleósido de la reversa transcriptasa o un inhibidor de proteasas; $\mathrm{VIH}=$ virus de inmunodeficiencia humana; CV=carga viral.

\section{Figura 2}

Uso de TARGA en gestantes infectadas por el VIH y transmisión vertical del virus. Cuba 2003-2008.
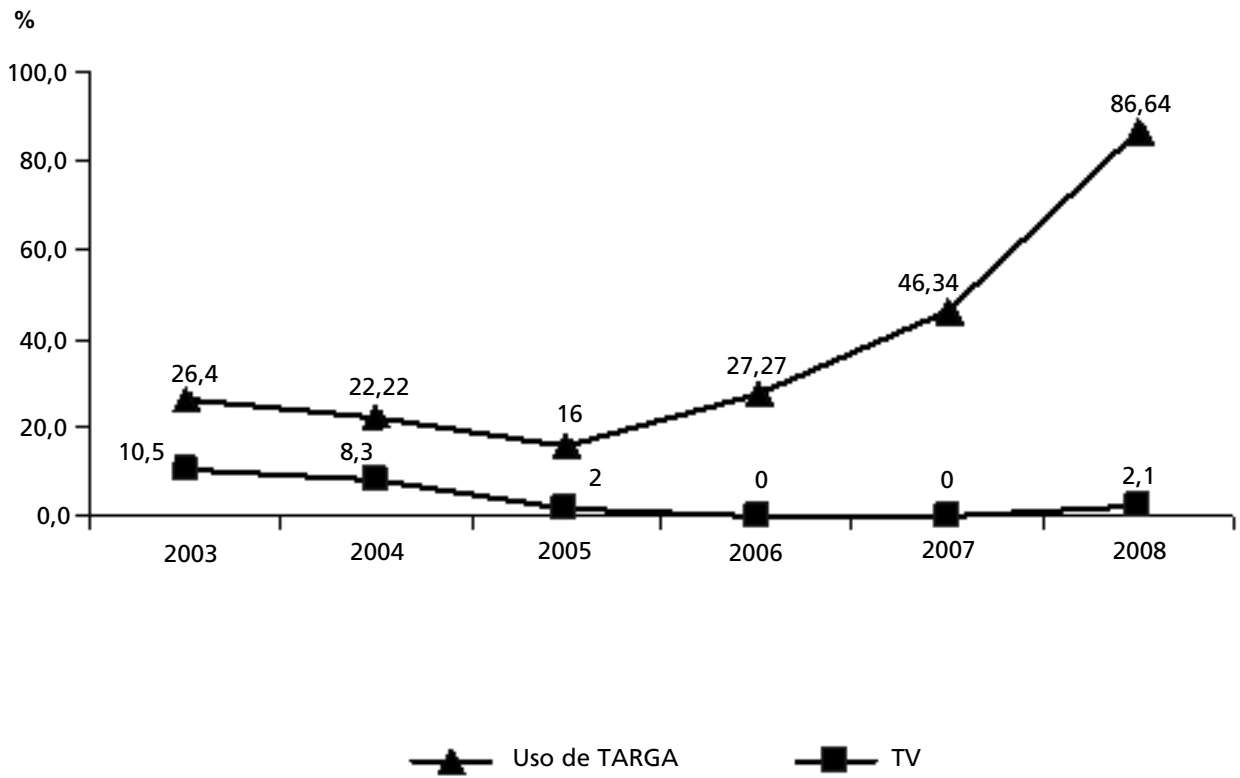

TARGA = terapia con tres antirretrovirales con al menos un inhibidor no nucleósido de la reversa transcriptasa o un inhibidor de proteasas; VIH= virus de inmunodeficiencia humana; TV = transmisión vertical. 
Transmisión vertical del VIH según tipo de tratamiento usado en la madre. Cuba 1989-2008.

\begin{tabular}{lcccccc}
\hline & \multicolumn{2}{c}{ Infectados $(\mathbf{n}=\mathbf{1 6})$} & No infectados $(\mathrm{n}=\mathbf{3 0 3})$ & & TV \% & RP (IC95\%) \\
\cline { 2 - 5 } Tratamiento & $\mathrm{n}$ & $\%$ & $\mathrm{n}$ & $\%$ & & \\
\hline TARGA* $^{*}$ & 2 & 12,50 & 86 & 28,38 & 2,27 & 1,00 \\
Biterapia & 0 & 0,00 & 3 & 0,99 & 0,00 & 0,00 \\
AZT sola & 7 & 43,75 & 176 & 58,09 & 3,83 & $1,68(0,36-7,36)$ \\
ninguno & 7 & 43,75 & 38 & 12,54 & 15,56 & $6,84(1,48-31,80)$ \\
\hline
\end{tabular}

* nivel de referencia; Homogeneidad entre niveles $\chi^{2}=12,5462 ; \mathrm{gl}=3 ; p=0,006$; Tendencia lineal $\chi^{2}=5,7571 ; \mathrm{gl}=1 ;$ $p=0,017 ; \mathrm{VIH}=$ virus de inmunodeficiencia humana; TV= transmisión vertical; TARGA = terapia con tres antirretrovirales con al menos un inhibidor no nucleósido de la reversa transcriptasa o un inhibidor de proteasas; RP=razón de prevalencia; IC=intervalo de confianza.

\section{Discusión}

El perfeccionamiento del programa de prevención cubano influye en la disminución de la transmisión vertical del VIH. En los años 2005-2008 se completa el uso de las tres partes de AZT al contar con la presentación endovenosa y se le da más valor al estado virológico de la gestante para definir el tratamiento antirretroviral, sobre todo en el año 2008 , donde más del $85 \%$ de las gestantes seropositivas al VIH recibieron TARGA.

La incorporación paulatina de medidas preventivas de mayor impacto se relaciona con la disminución de la transmisión vertical en muchas regiones. En Bélgica la transmisión fue de $10 \%$ en los años 1986-1993, de $8,1 \%$ en $1994-1998$ y de $4,7 \%$ en 1999-2002 9; en Cataluña, España la tasa de infección disminuyó significativamente del período 1987 1993 al $1997-2003(20,4 \%$ vs $3,5 \% ; p<0,01) 10 \mathrm{e}$ Israel durante los años 2000-2002 tuvo una tasa de transmisión de $6,1 \%$ y descendió a $0,7 \%$ en el período 2003-2005.11

En los países del continente americano también se aprecia ese comportamiento: Chile reportó una transmisión antes de instaurar el protocolo de prevención del $35,4 \%$ y posteriormente del $2,4 \% 12$ y en diferentes regiones de Brasil se reporta disminución significativa de la infección de la descendencia con el perfeccionamiento del programa preventivo (Belo Horizonte 20\% en 1998 vs 3\% 2005,13 São Paulo $16 \%$ en 1995 vs 2,4\% en 200214).

Se reporta que el uso de TARGA en gestantes infectadas por el VIH disminuye significativamente la transmisión vertical de la infección al compararla con el uso de AZT y/o nevirapina en dosis única (Botswana $0,4 \%$ vs 5,5\%; $p<0,00115$ y República Dominicana 3,3\% vs 15,4\%; respectivamente ${ }^{16}$ ).

Al usarse un esquema de tratamiento de mayor actividad muchas investigaciones demuestran que disminuye la transmisión del VIH a la descendencia como la realizada en el Instituto Materno perinatal de Lima, Perú, donde la transmisión vertical en el período que solo se usaba AZT fue de $15 \%$ y cuando se indicaba TARGA a todas las gestantes fue de 4\% ${ }^{17}$ y la conducida en Ciudad de Campinas São Paulo, donde en el periodo que se usaba AZT en la madre y el niño (1995-1996) la infección de la descendencia fue del $25,7 \%$ y en el período donde se usaban múltiples agentes antirretrovirales y la operación cesárea fue de $2,9 \%$, destacándose un incremento del uso de terapia antirretroviral combinada de $0 \%$ en el año 1990 a 46,4\% en el 2000 y no transmisión de la infección en las mujeres tratadas con múltiples drogas. 18

En la investigación que se presenta se observaron dos niños infectados en el grupo con antecedentes de uso de TARGA en sus madres $(2,3 \%)$; en uno de ellos el diagnóstico de la infección de su madre se realizó antes del embarazo y existían evidencias de fracaso del tratamiento antirretroviral y no se realizaron cambios en el tratamiento y en el otro el diagnóstico de su madre se realizó cerca de las 35 semanas de embarazo y se produjo su nacimiento tres semanas después del inicio del tratamiento antirretroviral.

La duración del tratamiento antirretroviral antenatal se relaciona con la transmisión vertical del VIH. La terapia antirretroviral insuficiente (no tratamiento antenatal o solo de uno a 13 días de tratamiento con TARGA) se asocia fuertemente con la transmisión vertical del VIH $(7,4 \% \text { vs } 1,1 \%)^{19} \mathrm{y}$ una investigación canadiense reporta que con TARGA la infección de la descendencia es de un $1 \%$ y que disminuye al $0,4 \%$ si se cumple por un período de cuatro semanas o más. 20

En Cuba actualmente toda las gestantes infec- 
tadas por el VIH reciben TARGA y en las mujeres que se diagnostican como infectadas por el VIH en etapas tardías del embarazo, se incluye en la combinación un inhibidor de proteasas como lopinavir/ritonavir; el país cuenta con la tecnología para realizar estudios de resistencia genotípica desde el año 2009, a mujeres con evidencias de fallo virológico, por niveles de carga viral plasmática persistentemente detectable o con supresión viral subóptima.

\section{Referências}

1. CDC. Achievements in Public Health: reduction in perinatal transmission of HIV infection - United States, 1986-2005. MMWR. 2006; 55(21): 592-8 [visitado el 30 de junio del 2006]. Disponible en: http: //www cdc.gov/mmwr/preview/ mmwrhtm/mm5221a3.htm

2. Guidelines for the use of antiretroviral agents in HIV-1infected adults and adolescents. [visitado el 18 de mayo del 2009]. Disponible en: http:// www.aidsinfo.nih.gov/guidelines/guidelinesDetail.aspx?menultem=Guidelines\&Search $=$ Off \&GuidelineID $=7 \&$ Class ID $=1$

3. Bartlett J, Sax PE, Young B. Treatment Update. Faculty Responses to Additional Questions on Initiating Antiretroviral Therapy. [visitado el 24 de Junio del 2009]. Disponible en: http:// www.list.clinicaloptions.com/ t/15827/103465/4643/0/.

4. González- Núñez I. SIDA en el niño por transmisión vertical en Cuba [tesis]. Ciudad Habana: IPK; 2005.

5. González- Núñez I, Díaz -Jidy M. Transmisión vertical del VIH/SIDA. En: Rodríguez Elías B, editora. Pautas Cubanas para el tratamiento antirretroviral en los pacientes con VIH/SIDA. Ciudad Habana: MINSAP; 2004. p. 47-64.

6. González- Nuñez I, Manuel Díaz -Jidy M, Verdasquera Corcho D, Pérez- Ávila J. Programa de prevención y control de la transmisión vertical del VIH en Cuba. Enero de 1986 - diciembre de 2007. Rev Chil Infect. 2010; 27: 320-6.

7. Connor EM, Sperling RS, Gelber R, Kiselev P, Scott G, O'Sullivan MJ, VanDyke R, Bey M, Shearer W, Jacobson RL, Jimenez E, O'Neill E, Bazin B, Delfraissy JF, Culnane M, Coombs R, Elkins M, Moye J, Stratton P, Balsley J. Reduction of maternal-infant transmission of human immunodeficiency virus type 1 with zidovudine treatment. Pediatric AIDS Clinical Trials Group Protocol 076 Study Group. N Engl J Med. 1994; 331: 1173-80.

8. Xunta de Galicia y Organización Panamericana de Salud. Programa para análisis epidemiológico de datos tabulados (EPIDAT). Versión 3.1. Enero 2006. Disponible en: http://www.dxsp.sergas.es

9. Goetghebuer T, Haelterman E, Marvillet I, Barlow P, Hainaut M, Salameh A, Ciardelli R, Gerard M, Levy J. Vertical transmission of HIV in Belgium: a 1986-2002 retrospective analysis. Eur J Pediatr. 2009; 168: 79-85.

10. Rojano X, Almeda Ortega J, Sánchez Ruiz E, Fortuny C, Bertrán JM, Mur Sierra A, Rodrigo Gonzalo de Liria C, Casabona I, Barbarà J; por el Grupo de estudio NENEXP.
Cuba a pesar de ser un país subdesarrollado se comporta en esta temática como los países industrializados porque existe el conocimiento científico necesario para afrontar la atención de las mujeres viviendo con VIH, sus hijos y sus familias. Una prueba de ello es la disminución de la transmisión vertical, en el último período que abarca esta investigación, que coincide con el perfeccionamiento del programa de prevención cubano.

Trends of HIV mother-to-child transmission in Catalonia, Spain, between 1987 and 2003. Med Clin (Barc). 2007; 129: 487-93

11. Agmon-Levin N, Elbirt D, Asher I, Torten D, Cohen Y, Gradestein S, Werner B, Turner D, Chowers M, Gottesman G, Maayan S, Risenberg K, Levi I, Sthoeger Z. Prevention of human immunodeficiency virus mother-to-child transmission in Israel. Int J STD \& AIDS. 2009; 20: 473-6.

12. Zamorano J, Hirsch T. Infección por virus de inmunodeficiencia humana, embarazo y deseo de reproducción. Comité de SIDA Pediátrico Sociedad Chilena de Pediatría. Rev Chil Infect. 2004; 21: 208-12.

13. Kakehasi FM, Pinto JA, Romanelli RM, Carneiro M, Cardoso CS, Tavares Mdo C, Melo VH, Aguiar RA. Determinants and trends in perinatal human immunodeficiency virus type 1 (HIV-1) transmission in the metropolitan area of Belo Horizonte, Brazil: 1998 - 2005. Mem Inst Oswaldo Cruz. 2008; 103(4): 351-7.

14. Matida LH, da Silva MH, Tayra A, Succi RC, Gianna MC, Gonçalves A, de Carvalho HB, Hearst N. Prevention of mother-to-child transmission of HIV in São Paulo State, Brazil: an update. AIDS. 2005; 19 (Suppl. 4): S37-41.

15. Dryden-Peterson S, Jayeoba O, Hughes MD, Jibril $\mathrm{H}$, Keapoletswe K, Tlale J, Modise TA, Asmelash A, Moyo S, van Widenfelt E, Makhema J, Essex M, Shapiro RL, Lockman S. Highly Active Antiretroviral Therapy versus Zidovudine for Prevention of Mother-to-Child Transmission in a Programmatic Setting, Botswana. J Acquir Immune Defic Syndr. 2011; 58: 353-2.

16. Schmidt NC, Roman-Pouriet J, Fernandez AD, Beck-Sagué CM, Leonardo-Guerrero J, Nicholas SW. Costs and benefits of multidrug, multidose antiretroviral therapy for prevention of mother-to-child transmission of HIV in the Dominican Republic. Int J Gynaecol Obstet. 2012;116 (3): 219-22.

17. Velásquez C. Results of the implementation of three national guidelines for the prevention of HIV vertical transmission in Instituto Nacional Materno Perinatal. Lima, Perú. Rev Perú Med Exp Salud Pública. 2011; 28 (3): 4926.

18. Amaral E, Assis-Gomes F, Milanez H, Cecatti JG, Vilela MM, Pinto E Silva JL. Timely implementation of interventions to reduce vertical HIV transmission: a successful experience in Brazil. Rev Panam Salud Pública. 2007; 21 (6): 357-64. 
19. European Collaborative Study in EuroCoord. Insufficient antiretroviral therapy in pregnancy: missed opportunities for prevention of mother-to-child transmission of HIV in Europe. Antivir Ther. 2011; 16: 895-903.

Recebido em 22 de janeiro de 2013

Versão final apresentada em 4 de março de 2013

Aprovado em 2 de abril de 2013
20. Forbes JC, Alimenti AM, Singer J, Brophy JC, Bitnun A, Samson LM, Money DM, Lee TC, Lapointe ND, Read SE, Canadian Pediatric AIDS Research Group (CPARG). A national review of vertical HIV transmission. AIDS. 2012; 26 (6): 757-63. 